\title{
Cytotoxic $T$ lymphocyte associated antigen 4 expression predicts poor prognosis in luminal B HER2-negative breast cancer
}

\author{
GAOCHEN LAN ${ }^{1 *}$, JIE LI $^{2 *}$, QIAOMEI WEN ${ }^{3}$, LIN LIN $^{2}$, LIBIN CHEN $^{4}$, LIYU CHEN $^{5}$ and XI CHEN ${ }^{2}$ \\ ${ }^{1}$ Department of Oncology, Fujian Tingzhou Hospital, Longyan, Fujian 366300; \\ ${ }^{2}$ Department of Oncology, Fuzhou General Hospital of Nanjing Military Command, Fuzhou, Fujian 350025; \\ ${ }^{3}$ Department of Traditional Chinese Medicine, Lihe Traditional Chinese Medicine Policlinic, Xiamen, Fujian 361000; \\ ${ }^{4}$ Department of Emergency, Jinjiang Hospital of Traditional Chinese Medicine, Quanzhou, Fujian 362200; \\ ${ }^{5}$ Department of Pathology, Affiliated Hospital of Putian College, Putian, Fujian 351100, P.R. China
}

Received July 5, 2017; Accepted December 8, 2017

DOI: 10.3892/ol.2018.7991

\begin{abstract}
Cytotoxic T lymphocyte associated antigen 4 (CTLA-4) serves an important role in inhibiting anti-tumor immune response in the majority of solid tumors. However, a limited number of studies reported the function of CTLA-4 in luminal B HER2-negative breast cancer. Immunohistochemistry was performed to evaluate the expression of tumor and interstitial CTLA-4 in luminal B HER2-negative breast cancer tissues. The percentage of patients with tumor and interstitial CTLA- $4^{+}$ was $41.2 \%$ (42/102) and $46.1 \%$ (47/102), respectively. There was a positive association between tumor CTLA-4 expression and interstitial CTLA-4 expression $(\mathrm{P}<0.05)$. The disease-free survival (DFS) of the tumor CTLA- $4^{+}$group was significantly shorter compared with patients with tumor CTLA-4- (mean, 89.070 vs. 39.022 months; $\mathrm{P}<0.0001)$. Additionally, the DFS of interstitial CTLA- $4^{+}$group was shorter compared with the interstitial CTLA-4- group (mean, 85.526 vs. 46.574 months; $\mathrm{P}<0.0001)$. Tumor and interstitial CTLA-4 expression may have prognostic predicting value in luminal B HER2-negative breast cancer. The present study may provide the basis for the use of a CTLA-4 blocker in patients with luminal B HER2-negative breast cancer.
\end{abstract}

\section{Introduction}

Breast cancer is the second largest cause of mortality in women worldwide $(1,2)$. Compared with western countries, the incidence in China has increased greatly since the 1990s $(3,4)$.

Correspondence to: Dr Xi Chen, Department of Oncology, Fuzhou General Hospital of Nanjing Military Command, 156 Xierhuan Northern Road, Fuzhou, Fujian 350025, P.R. China

E-mail: xichen2017sci@sina.com

*Contributed equally

Key words: cytotoxic T lymphocyte associated antigen 4, luminal B HER2-negative breast cancer, prognosis
Breast cancer is a heterogeneous disease, with five molecular subtypes, including luminal A, luminal B HER2-negative, luminal B HER2-positive, HER2-positive and triple negative. Furthermore, the incidence, treatment and prognosis vary greatly among these five molecular subtypes (5).

A recent epidemiological study demonstrated that the luminal B HER2-negative breast cancer had the highest incidence among these five molecular subtypes, and $>40 \%$ patients are Chinese women (6). Although luminal B HER2-negative breast cancer expresses hormone receptors, the curative effect of hormone therapy and conventional chemotherapy is unsatisfactory (7). Therefore, it is important to identify new targets for the treatment of luminal B HER2-negative breast cancer.

Cytotoxic T lymphocyte associated antigen 4 (CTLA-4), a CD28 homologue, consists of a short cytoplasmic tail, a signal peptide, a transmembrane domain and a cellular extracellular ligand-binding domain (8). CTLA-4 can be a competitive inhibitor for CD28, where it binds to the ligands; CD80 or CD86, resulting in inhibition of $\mathrm{T}$ cell activation and raising the response threshold of T cells (9). In fact, CTLA-4 has a stronger binding affinity with the two ligands compared with CD28, thus high expression of CTLA-4 may lead to inhibition of anti-tumor immune response (8). CTLA-4 may be expressed on the surface of $\mathrm{T}$ cells (10). However, there are some reports supporting the notion that CTLA-4 is also expressed on non-T cells, such as solid tumors (11-14). A recent study demonstrated that patients overexpressing CTLA-4 in esophageal cancer cells tend to have a poor prognosis (15).

Recent studies have also demonstrated that CTLA-4 is overexpressed in breast cancer cells $(10,11)$. However, the association between CTLA-4 expression and prognosis in breast cancer patients is not fully elucidated, particularly for luminal B HER2-negative breast cancer. Therefore, the present study aimed to answer such questions and provide a basis for new therapeutic targets for the treatment of luminal B HER2-negative breast cancer.

\section{Materials and methods}

Patients. A total of 102 cases of patients with stage I-III luminal B HER2-negative breast cancer who underwent radical 
surgery between January 2008 and December 2012 at Fuzhou General Hospital of Nanjing Military Command (Fuzhou, China) were selected for the present study. The study protocol was approved by the Medical Ethics Committee of the Fuzhou General Hospital. The clinicopathological characteristics of 102 patients were collected, which included age, histological grade, lymph node, menopausal status, tumor size and stage. The selection of clinicopathological characteristics was determined by the recurrence risk factors, which are recommended by the breast cancer prognosis guideline (16). The inclusion criteria were as follows: (i) Pathologically confirmed diagnosis of luminal B HER2-negative breast cancer according to the St Gallen International Expert Consensus 2013 (17); (ii) no chemotherapy or hormone therapy prior to surgery; (iii) paraffin-embedded specimens of tumor tissues were available, (iv) informed consent was obtained; (v) follow-up was available. The median follow-up time was 43.5 months (range, 1-96 months), which included 28 patients where metastasis or local recurrence was present. The disease-free survival (DFS) rate was $72.5 \%$. The median DFS rate was not obtained.

Immunohistochemistry (IHC). The surgical resection specimens of 102 patients were fixed with formalin and embedded by paraffin. Continuous paraffin section (thickness, $4 \mu \mathrm{m}$ ) were deparaffinized using $100 \%$ xylene, $100 \%$ ethanol, $95 \%$ ethanol and $80 \%$ ethanol. The tissue sections were used for antigen retrieval by high-pressure with $0.01 \mathrm{~mol} / \mathrm{l}$ ethylenediaminetetraacetic acid, and endogenous peroxidase activity was blocked by hydrogen peroxide at room temperature for $10 \mathrm{~min}$. The tissue sections were washed in PBS three times, and then primary antibodies anti-CTLA-4 $\operatorname{IgG}$ (dilution, 1:200; catalog no. bs-1179R; Beijing Biosynthesis Biotechnology Co., Ltd., Beijing, China) and anti-Ki-67 antibody (dilution, 1:200; catalog no. bs-23105R; Beijing Biosynthesis Biotechnology Co., Ltd.) were added to the tissue sections for $60 \mathrm{~min}$ at room temperature. Subsequently, the tissue sections were washed with PBS three times and incubated with EliVision ${ }^{\mathrm{TM}}$ plus Polyer horseradish peroxidase (Mouse/Rabbit) IHC kit (ready-to-use dilution; catalog no. KIT 9901; Fuzhou Maixin Biotechnology Co. Ltd., Fuzhou, China) at room temperature for $30 \mathrm{~min}$. Subsequently, the tissue sections were stained with 3,3-diaminobenzidine (Fuzhou Maixin Biotechnology Co. Ltd.) at room temperature for $3 \mathrm{~min}$. The sections were counterstained with hematoxylin at room temperature for $15 \mathrm{sec}$, and the slides were counterstained with neutral resin for subsequent observation with an optical microscope (magnification, x100).

Evaluation of IHC staining. A total of two independent pathologists, who were blinded to patient characteristics and clinical outcomes, evaluated results of IHC staining. The expression of CTLA-4 was evaluated with the percentage and intensity of positive tumor cells. The scores of percentage of positive tumor cells were recorded as: 0 (0-25\%), 1 (26-50\%), 2 (51-75\%) or 3 (76-100\%). The scores of intensity of positive tumor cells were recorded as: 0 (negative), 1 (weak), 2 (moderate) or 3 (strong). The scores for percentage and intensity were multiplied together to attain a final histochemical score (H-score). Then, the mean $\mathrm{H}$-score was calculated from all patient samples and determined as CTLA- 4 positivity (tumor CTLA- $4^{+}$). The CTLA-4 positivity in interstitial lymphocytes (interstitial CTLA $-4^{+}$), which was in interstitial areas adjacent to tumor nests, was evaluated as aforementioned. Ki-67 was negative if there was less than $14 \%$ of nuclei staining and positive if $\geq 14 \%$, in accordance with previous studies $(17,18)$.

Statistical analysis. The data was analyzed using SPSS (version 16.0; SPSS, Inc., Chicago, IL, USA). The association between CTLA-4 expression and various clinicopathologic parameters were analyzed by the $\chi^{2}$ test. The survival probability was estimated by the Kaplan-Meier test, and the statistical significance was performed using the log-rank test. The multivariate analysis for clinicopathological characteristics features was analyzed using the Cox regression model. All tests were two sided. $\mathrm{P}<0.05$ was considered to indicate a statistically significant difference.

\section{Results}

CTLA-4 expression in luminal B HER2-negative breast cancer tissues and interstitial tissues. To investigate the function of CTLA-4 in progression of luminal B HER2-negative breast cancer, IHC was conducted to detect the expression of CTLA-4 in tumor and interstitial tissues. CTLA-4 was expressed in the cell membrane and cytoplasm of $\mathrm{T}$ lymphocytes and tumor cells (Fig. 1A and B, respectively). The percentage of patients with tumor CTLA- $4^{+}$was $41.2 \%$ (42/102), while the percentage of patients with interstitial CTLA- $4^{+}$was $46.1 \%$ (47/102; Fig. 1C). In addition, to investigate whether there is an association between the expression of tumor and interstitial CTLA- 4 , the $\chi^{2}$ test was used to demonstrate that there was a strong positive association between tumor and interstitial CTLA-4 expression $(\mathrm{P}<0.05)$.

Association between CTLA-4 expression and clinicopathological characteristics. The $\chi^{2}$ test was performed to examine the association between CTLA-4 expression and clinicopathological characteristics in luminal B HER2-negative breast cancer tissues. However, neither the expression of tumor CTLA-4 nor interstitial CTLA-4 was associated with any clinical characteristics of the patients in the present cohort, including age, histological grade, lymph node, menopausal status, tumor size and stage and $\mathrm{Ki}-67$ expression (all $\mathrm{P}>0.05$; Table I).

Association between tumor/interstitial CTLA-4 expression and clinical outcomes of patients with luminal $B$ HER2-negative breast cancer tissues. The Kaplan-Meier method was performed to examine the association between the expression of tumor/interstitial CTLA-4 and the survival of patients with luminal B HER2-negative breast cancer. In the present study, the DFS rate of patients with tumor CTLA- $4^{+}$ was significantly shorter compared with patients with tumor CTLA-4- (mean, 89.070 vs. 39.022 months; P<0.0001; Fig. 2A). Additionally, the DFS rate of the patients with interstitial CTLA $-4^{+}$was considerably shorter compared with patients with interstitial CTLA-4- (mean, 85.526 vs. 46.574 months; $\mathrm{P}<0.0001$; Fig. 2B). Furthermore, the multivariate cox regression analysis demonstrated that tumor CTLA- $4^{+}$was an independent predictor of shorter DFS when factors, including 
Table I. Association between CTLA-4 expression and clinicopathological characteristics.

\begin{tabular}{|c|c|c|c|c|c|c|c|}
\hline \multirow{2}{*}{$\begin{array}{l}\text { Clinicopathological } \\
\text { characteristics }\end{array}$} & \multirow[b]{2}{*}{$\mathrm{n}$} & \multicolumn{2}{|c|}{$\begin{array}{l}\text { Tumor } \\
\text { CTLA-4 }\end{array}$} & \multirow[b]{2}{*}{ P-value } & \multicolumn{2}{|c|}{$\begin{array}{l}\text { Interstitial } \\
\text { CTLA-4 }\end{array}$} & \multirow[b]{2}{*}{ P-value } \\
\hline & & $(+)$ & $(-)$ & & $(+)$ & $(-)$ & \\
\hline Age (years) & & & & 0.099 & & & 0.845 \\
\hline$<35$ & 8 & 6 & 2 & & 5 & 3 & \\
\hline$\geq 35$ & 94 & 36 & 58 & & 49 & 45 & \\
\hline Menopausal status & & & & 0.868 & & & 0.560 \\
\hline Premenopausal & 52 & 21 & 31 & & 29 & 23 & \\
\hline Postmenopausal & 50 & 21 & 29 & & 25 & 25 & \\
\hline Histological grade & & & & 0.422 & & & 0.148 \\
\hline I & 16 & 6 & 10 & & 11 & 5 & \\
\hline II & 58 & 27 & 31 & & 32 & 26 & \\
\hline III & 28 & 9 & 19 & & 11 & 17 & \\
\hline Tumor size $(\mathrm{cm})$ & & & & 0.636 & & & 0.243 \\
\hline$\leq 2$ & 49 & 19 & 30 & & 23 & 26 & \\
\hline$>2$ & 53 & 23 & 30 & & 31 & 22 & \\
\hline Lymph node & & & & 0.332 & & & 0.161 \\
\hline Negative & 52 & 19 & 33 & & 24 & 28 & \\
\hline Positive & 50 & 23 & 27 & & 30 & 20 & \\
\hline Tumor stage & & & & 0.298 & & & 0.114 \\
\hline I & 29 & 10 & 19 & & 11 & 18 & \\
\hline II & 47 & 18 & 29 & & 26 & 21 & \\
\hline III & 26 & 14 & 12 & & 17 & 9 & \\
\hline Ki-67 & & & & 0.884 & & & 0.376 \\
\hline$<14$ & 9 & 3 & 6 & & 3 & 6 & \\
\hline$\geq 14$ & 93 & 39 & 54 & & 51 & 42 & \\
\hline
\end{tabular}

CTLA-4, cytotoxic T lymphocyte-associated antigen 4.

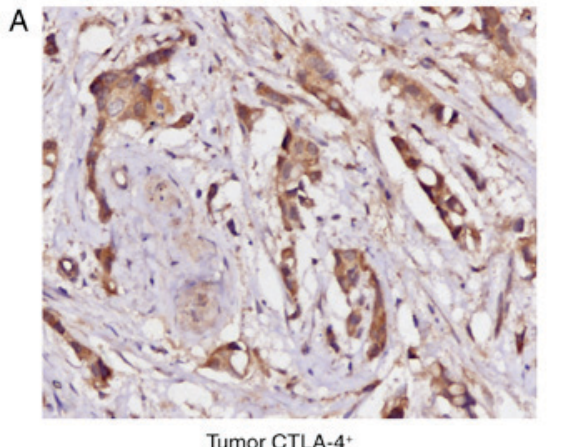

Tumor CTLA-4 ${ }^{+}$

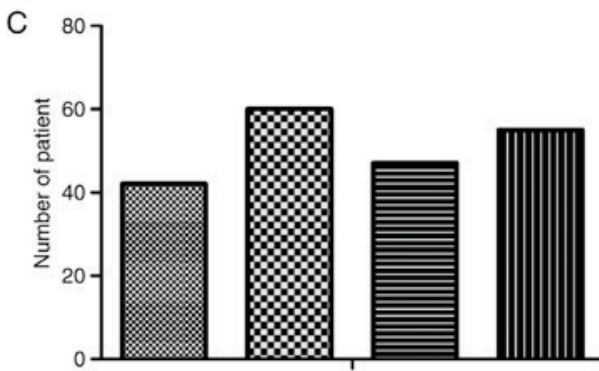

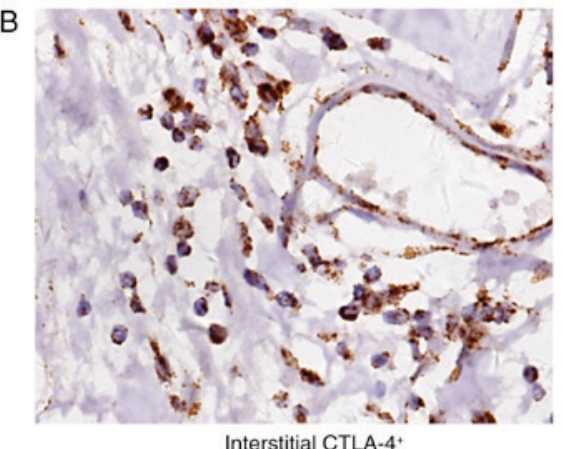

网 Tumor CTLA-4+

B. Tumor CTLA-4

E Interstitial CTLA-4

III Interstitial CTLA-4

Figure 1. CTLA-4 ${ }^{+}$expression in (A) tumor and (B) interstitial tissue (magnification x100). (C) Quantification of CTLA-4 ${ }^{+}$and CTLA-4 ${ }^{-}$as analyzed by IHC. CTLA-4, cytotoxic T lymphocyte-associated antigen 4; IHC, immunohistochemistry. 
Table II. Multivariate analysis of patient survival.

\begin{tabular}{lclr}
\hline $\begin{array}{l}\text { Clinicopathological } \\
\text { characteristics }\end{array}$ & HR & \multicolumn{1}{c}{ 95\% CI } & P-value \\
\hline Tumor CTLA-4 & 0.058 & $0.015-0.224$ & $<0.001$ \\
Ki-67 & 4.406 & $0.528-36.756$ & 0.171 \\
Tumor stage & 2.769 & $1.181-6.488$ & 0.019 \\
Lymph node & 0.425 & $0.128-1.413$ & 0.163 \\
Tumor size & 0.842 & $0.349-2.034$ & 0.702 \\
Years & 2.337 & $0.471-11.591$ & 0.299 \\
Menopausal status & 1.420 & $0.610-3.308$ & 0.416 \\
Histological grade & 1.086 & $0.559-2.111$ & 0.807 \\
Interstitial CTLA-4 & 1.413 & $0.430-4.637$ & 0.569 \\
\hline
\end{tabular}

CTLA-4, cytotoxic T lymphocyte-associated antigen 4; CI, confidence interval; HR, hazard ratio.
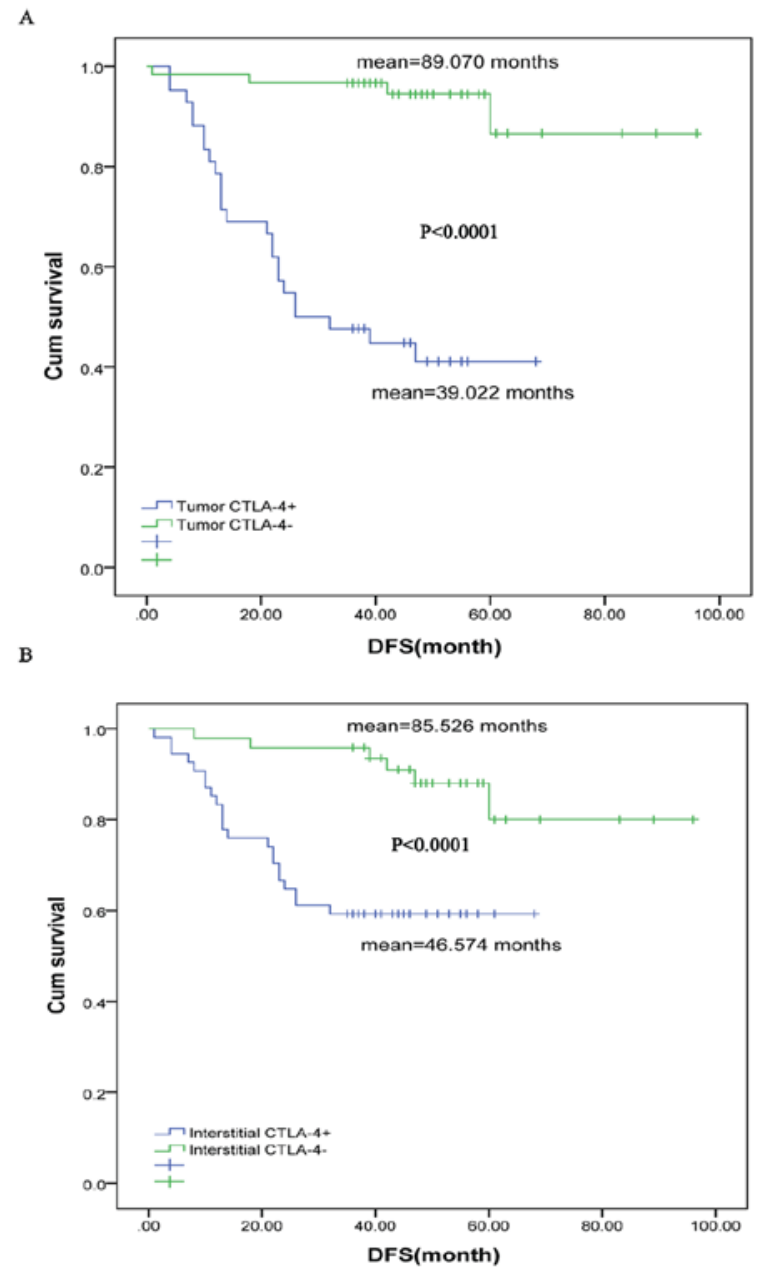

Figure 2. Kaplan-Meier analysis on the expression of (A) tumor and (B) interstitial CTLA and prognosis of breast cancer patients. CTLA-4, cytotoxic T lymphocyte-associated antigen 4; Cum, cumulative; DFS, disease-free survival.

age, menopausal status, histological grade, tumor size, the number of lymph nodes, clinical stage, $\mathrm{Ki}-67$ and interstitial CTLA-4, are controlled ( $\mathrm{P}<0.001$; Table II).

\section{Discussion}

Tumor-derived immune deregulation is a common characteristic in the majority of solid tumors, particularly for breast cancer (10). The immunosuppressive microenvironment primarily includes cytokines and immune checkpoint molecules, which leads to the blocking of anti-tumor immunity (19-21). One of these immune checkpoint molecules is the cytotoxic T lymphocyte antigen 4 (CTLA-4; CD152), a CD28 homologue which has two common ligands with CD28: B7-1 (CD80) and B7-2 (CD86). Notably, CTLA-4 has a stronger binding affinity with these two ligands compared with CD28 (8). The investigation of CTLA-4 is crucial for understanding tumor-derived immune deregulation and may give rise to novel immune therapy for breast cancer.

Previous studies have indicated that CTLA- 4 was overexpressed in breast cancer cells. However, these studies did not investigate the function of CTLA-4 in different types of breast cancer, particularly luminal B HER2-negative breast cancer, which has a high incidence in Chinese women (11). In the present study, the expression of CTLA- 4 was detected by IHC, and $41.2 \%(42 / 102)$ and $46.1 \%(47 / 102)$ were patients with tumor and interstitial CTLA $-4^{+}$, respectively. Furthermore, the association between tumor CTLA-4 and interstitial CTLA-4 was assessed. It was demonstrated that there was a positive association of tumor CTLA- 4 and interstitial CTLA-4. Subsequently, a $\chi^{2}$ test was performed to analyze the association between the expression of CTLA-4 and clinicopathological characteristics of the selected patients. However, the results demonstrated that the expression of neither tumor nor interstitial CTLA-4 was associated with any of the clinical characteristics assessed, including age, menopausal status, histological grade, tumor size, lymph node, tumor stage and $\mathrm{Ki}-67$ expression level.

Previous studies reported that patients exhibiting positive tumor CTLA-4 expression had a better prognosis in NSCLC and gastric cancer $(12,13)$. However, previous studies also demonstrated that CTLA-4 was a poor prognosis factor in esophageal carcinoma and breast cancer $(10,19)$. Therefore, we hypothesized that CTLA-4 expression may be associated with the prognosis of patients with luminal B HER2-negative breast cancer. Furthermore, tumor CTLA- $4^{+}$was an independent risk factor for the prognosis of patients with breast cancer.

The results of a clinical trial for a CTLA-4 blocker, tremelimumab, for the treatment of breast cancer demonstrated that in the majority of patients, peripheral blood immune function was improved (22). Therefore, CTLA-4 may be a novel target for the treatment of luminal B HER2-negative breast cancer. However, the study by Vonderheide et al (22) demonstrated that there was no association between peripheral blood immune function and clinical outcomes (22). Tumor microenvironment may serve a greater role than immune status of the peripheral blood in anti-tumor immunotherapy. Tumor microenvironment may be taken into account when CTLA- 4 becomes an immunotherapy target for breast cancer.

The present study has some limitations. More patients are required in future studies in order to confirm that CTLA-4 can be a biomarker for assessing the clinical outcomes of anti-CTLA-4 treatment in luminal B HER2-negative breast cancer. Furthermore, the association between tumor and 
interstitial CTLA-4, and the mechanism by which CTLA-4 enters the tumor cells were not investigated

In conclusion, the present study demonstrated a positive association between the expression of tumor and interstitial CTLA-4, which was associated with poor prognosis in luminal B HER2-negative breast cancer. The present study may provide novel therapeutic targets for patients with luminal B HER2-negative breast cancer. However, further studies are required to confirm these findings.

\section{Competing interests}

The authors declare that they have no competing interests.

\section{References}

1. DeSantis C, Ma J, Bryan L and Jemal A: Breast cancer statistics, 2013. CA Cancer J Clin 64:52-62, 2014.

2. Siegel R, Miller K and Jemal A: Cancer statistics, 2015. CA Cancer J Clin 65: 5-29, 2015.

3. Chen W, Zheng R, Baade PD, Zhang S, Zeng H, Bray F, Jemal A, $\mathrm{Yu}$ XQ and He J: Cancer statistics in China, 2015. CA Cancer J Clin 66: 115-132, 2016.

4. Zhang ML, Huang ZZ and Zheng Y: Estimates and prediction on indidence, mortality and prevalence of breast cancer in China, 2008. Zhonghua Liu Xing Bing Xue Za Zhi 33: 1049-1051, 2012, (In Chinese).

5. Cancer Genome Atlas Network. Comprehensive molecular portraits of human breast tumors. Nature 490: 61-70, 2012.

6. Si W, Li Y, Han Y, Zhang F, Wang Y, Li Y, Linghu RX, Zhang X and Yang J: Epidemiological and clinicopathological trends of breast cancer in chinese patients during 1993 to 2013: A retrospective study. Medicine (Baltimore) 94: e820, 2015.

7. Tran B and Bedard PL: Luminal-B breast cancer and novel therapeutic targets. Breast Cancer Res 13: 221, 2011.

8. Grosso JF and Jure-Kunkel MN: CTLA-4 blockade in tumor models: An overview of preclinical and translational research. Cancer Immun 13:5, 2013.

9. Schwartz JC, Zhang X, Fedorov AA, Nathenson SG and Almo SC: Structural basis for co-stimulation by the human CTLA-4/B7-2 complex. Nature 410: 604-608, 2001.

10. Yu H, Yang J, Jiao S, Li Y, Zhang W and Wang J: Cytotoxic $\mathrm{T}$ lymphocyte antigen 4 expression in human breast cancer: Implications for prognosis. Cancer Immunol Immunother 64: 853-860, 2015.

11. Mao H, Zhang L, Yang Y, Zuo W, Bi Y, Gao W, Deng B, Sun J, Shao Q and Qu X: New Insights of CTLA-4 into its biological function in breast cancer. Curr Cancer Drug Targets 10: 728-736, 2010.
12. Salvi S, Fontana V, Boccardo S, Merlo DF, Margallo E, Laurent S, Morabito A, Rijavec E, Dal Bello MG, Mora M, et al: Evaluation of CTLA-4 expression and relevance as a novel prognostic factor in patients with non-small cell lung cancer. Cancer Immunol Immunother 61: 1463-1472, 2012.

13. Kim JW, Nam KH, Ahn SH, Park DJ, Kim HH, Kim SH, Chang H, Lee JO, Kim YJ, Lee HS, et al: Prognostic implications of immunosuppressive protein expression in tumors as well as immune cell infiltration within the tumor microenvironment in gastric cancer. Gastric Cancer 19: 42-52, 2016.

14. Laurent S, Carrega P, Saverino D, Piccioli P, Camoriano M, Morabito A, Dozin B, Fontana V, Simone R, Mortara L, et al: CTLA-4 is expressed by human monocyte-derived dendritic cells and regulates their functions. Hum Immunol 71: 934-941, 2010.

15. Zhang XF, Pan K, Weng DS, Chen CL, Wang QJ, Zhao JJ, Pan QZ, Liu Q, Jiang SS, Li YQ, et al: Cytotoxic T lymphocyte antigen-4 expression in esophageal carcinoma: Implications for prognosis. Oncotarget 7: 26670-26679, 2016.

16. Ehinger A, Malmström P, Bendahl PO, Elston CW, Falck AK, Forsare C, Grabau D, Rydén L, Stål O and Fernö M; South and South-East Swedish Breast Cancer Groups: Histological grade provides significant prognostic information in addition to breast cancer subtypes defined according to St Gallen 2013. Acta Oncol 56: 68-74, 2017.

17. Goldhirsch A, Winer EP, Coates AS, Gelber RD, Piccart-Gebhart M, Thürlimann B and Senn HJ; Panel members: Personalizing the treatment of women with early breast cancer: Highlights of the St Gallen international expert consensus on the primary therapy of early breast cancer 2013. Ann Oncol 24: 2206-2223, 2013

18. Prihantono $\mathrm{P}$, Hatta $\mathrm{M}$, Binekada $\mathrm{C}$, Sampepajung $\mathrm{D}$ Haryasena H, Nelwan B, Asadul Islam A and Nilawati Usman A: Ki-67 expression by immunohistochemistry and quantitative real-time polymerase chain reaction as predictor of clinical response to neoadjuvant chemotherapy in locally advanced breast cancer. J Oncol 2017: 6209849, 2017.

19. Emens LA: Breast cancer immunobiology driving immunotherapy: Vaccines and immune checkpoint blockade. Expert Rev Anticancer Ther 12: 1597-1611, 2012.

20. Koch MA, Thomas KN, Perdue NR, Smigiel KS, Srivastava S and Campbell DJ: T-bet(+) Treg cells undergo abortive Th1 cell differentiation due to impaired expression of IL-12 receptor $\beta 2$. Immunity 37: 501-510, 2012.

21. Yu HM, Yang JL, Jiao SC, Wang JD and Li Y: TGF- $\beta 1$ precursor and CD 8 are potential prognostic and predictive markers in operated breast cancer. J Huazhong Univ Sci Technolog Med Sci 34: $51-58,2014$

22. Vonderheide RH,LoRusso PM, Khalil M, Gartner EM, Khaira D, Soulieres D, Dorazio P, Trosko JA, Rüter J, Mariani GL, et al: Tremelimumab in combination with exemestane in patients with advanced breast cancer and treatment-associated modulation of inducible costimulator expression on patient $\mathrm{T}$ cells. Clin Cancer Res 16: 3485-3494, 2010. 